\title{
Does muscular activity related to vertical facial divergence influence the time needed for orthodontic extrusion of palatally impacted maxillary canines? A retrospective study
}

\author{
Michele Tepedino ${ }^{1}$, Maciej Iancu-Potrubacz ${ }^{1}$, Cristina Grippaudo ${ }^{2}$, Claudio Chimenti ${ }^{1}$, Giuseppina Laganà ${ }^{3}$ \\ ${ }^{1}$ Department of Biotechnological and Applied Clinical Sciences, University of L'Aquila, L'Aquila, Italy \\ ${ }^{2}$ Fondazione Policlinico Universitario A. Gemelli IRCCS, Roma-Università Cattolica del Sacro Cuore. Istituto di Clinica Odon- \\ toiatrica e Chirurgia Maxillo-facciale, Rome, Italy \\ ${ }^{3}$ Department of Clinical Sciences and Translational Medicine, University of Rome Tor Vergata, Rome, Italy
}

Correspondence:

Department of Biotechnological and Applied Clinical Sciences

University of L'Aquila

V.le S.Salvatore, Edificio Delta 6

67100 L'Aquila, $A Q$, Italy

m.tepedino@hotmail.it

Received: 17/07/2018

Accepted: 06/08/2018

\begin{abstract}
Tepedino M, Iancu-Potrubacz M, Grippaudo C, Chimenti C, Laganà G. Does muscular activity related to vertical facial divergence influence the time needed for orthodontic extrusion of palatally impacted maxillary canines? A retrospective study. J Clin Exp Dent. 2018;10(9):e869-75.

http://www.medicinaoral.com/odo/volumenes/v10i9/jcedv10i9p869.pdf
\end{abstract}

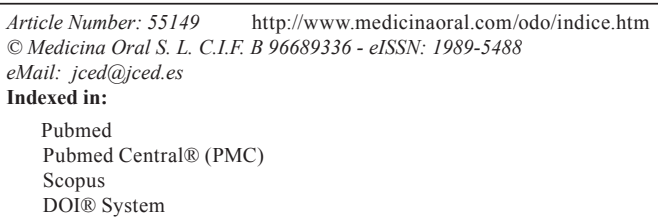

DOI System

\begin{abstract}
Background: The aim of the present study was to evaluate if the different muscular activity correlated to different degrees of facial divergence has an effect on the time needed to extrude a palatally impacted maxillary canine. Material and Methods: Twenty-six patients were retrospectively selected, all treated with a specific cantilever appliance that allows extrusion of the impacted canine applying a physiologic amount of force below $0.6 \mathrm{~N}$ in a predictable way. For all the patients, pre-treatment cephalometric tracings were used to evaluate facial divergence through the FMA angle, the angle between the maxillary and mandibular plane, and the angles between the occlusal plane and either the maxillary and mandibular plane. Linear bivariate regression was calculated to evaluate if facial divergence can predict the time needed for canine extrusion.

Results: The linear regression model was not able to predict extrusion time from variables explaining the facial divergence.

Conclusions: Palatally impacted maxillary canines can be treated with the application of physiologic extrusion force regardless of patients' facial divergence and muscular activity.
\end{abstract}

Key words: Impacted canines, cantilever, facial divergence, muscular activity.

\section{Introduction}

The impaction of maxillary canines can occur in nearly $2 \%$ of the population (1), more specifically $2.4 \%$ in the italian population (2), being caused by either genetic or environmental factors (3-5). When an alteration in the eruption pathway is early intercepted, an interceptive treatment comprising extraction of the deciduous canine(1) and rapid maxillary expansion (6), possibly anchored on deciduous teeth $(7,8)$, can be performed. Nevertheless, in many cases orthodontists have to mechanically erupt an impacted canine. In most of the cases, the impacted canine is palatally displaced $(85 \%$ of the cases), requiring a treatment that is usually complex and time-consuming (9). In fact, the treatment of a ma- 
locclusion that comprises an impacted tooth requires more time than a similar malocclusion without impaction (10), and needs a complex planification because the selection of the surgical technique, the modality of orthodontic traction, the arch space management, and the preparation of the anchorage should be carefully planned (11-13). All the enlisted factors should be addressed to achieve a satisfactory functional and aesthetical result, avoiding complications such as root resorption and loss of vitality of impacted or neighboring teeth (14-16). To improve the efficiency of orthodontic treatment of palatally impacted maxillary canines, several techniques and appliances have been proposed, including Kilroy springs, double archwires, powerchains, magnets, and cantilevers (17). Most of these systems, as highlighted in an article by Yadav et al. (18), produce high forces, around $2.5 \mathrm{~N}$, that exceed the recommended threshold of $0.6 \mathrm{~N}(14)$, resulting in a higher risk of complications. In two previous articles, we proposed a cantilever system that allows the use of light physiologic force in a predictable way, with the advantage of having a device made out of stainless steel $(19,20)$.

While it is known that orthodontic treatment is generally followed by a muscular and functional adaptation (2123 ), the effects of masticatory muscles associated with different kinds of tooth movements should be evaluated during treatment planning (24). For example, strong bite forces are observed in subjects with parallel jaws and augmented posterior face height, while weaker bite forces are associated with long-face patients (25), both in adults and children (26), and these aspects influences orthodontic tooth movements (27). In addition, since the device used for extrusion of maxillary impacted canines is located in the palatal vault, the effect of the tongue should be also considered: the tongue has the ability to deliver orthodontic forces and to move teeth (28), and different tongue and hyoid bone positions have been observed in hypodivergent and hyperdivergent subjects (29) that can result in different magnitude of tongue pressure (30).

The purpose of this study was to evaluate the effect of the different muscular and tongue patterns in hypodivergent and hyperdivergent patients on the time needed to extrude a palatally impacted canine with a device that predictably produces an amount of force around $0.6 \mathrm{~N}$. The null hypothesis was that facial divergence has no effect on the time needed to extrude the impacted tooth.

\section{Material and Methods}

The present research protocol was approved by the Internal Review Board of the University of L'Aquila (Protocol number 23169). The records of patients treated for the orthodontic extrusion of an impacted maxillary canines at the Dental clinic of the Department of Biotechnological and Applied Clinical Sciences, University of L'Aquila from January 2007 to January 2018 were retrospectively screened for the following inclusion criteria:

- Unilateral or bilateral canine impaction with a palatal displacement;

- Orthodontic traction performed with a calibrated amount of force through a previously described cantilever appliance (19);

- No failure of the traction (debonding of the canine's bracket, breakage of the ligature, etc.); reported in patient's clinical history that could have affected extrusion time;

- Absence of local or systemic conditions that could alter bone metabolism and tooth movement.

Sample size calculation (G*Power version 3.1.9.2, Universitat Dusseldorf, Germany) (31) revealed that for a linear bivariate regression with an $\alpha$ error of 0.05 , a power of 0.8 , and a calculated slope of 0.36 , a sample of 26 subjects was needed. Therefore, the first 26 subjects in chronological order that met the inclusion criteria were included in the study sample.

All patients had palatally impacted canines extruded with a specific device described in a previous work (19): the appliance was made of a $0.9 \mathrm{~mm}$ stainless steel transpalatal arch with a distal loop welded to two molar bands for the upper first molars, and a $0.6 \mathrm{~mm}$ stainless steel cantilever welded to the transpatal arch and then rolled around it to create 5 loops. The cantilever was pre-activated to have its end at approximately $15 \mathrm{~mm}$ from the point of force application on the crown of the impacted canine (Figs. 1,2). The singularity of the described appliance is that with such a configuration it is possible to predictably deliver to the impacted canine a physiologic force not exceeding $0.6 \mathrm{~N}$.

For all the selected patients, orthodontic extrusion time (from the moment when the cantilever was first tied to the impacted tooth until the moment when the cantilever was removed because the canine had reached the occlusal plane) was retrieved from the patient's record; in the case of bilateral impaction, the extrusion time of the two canines was averaged to have a single variable. In addition, pre-treatment lateral cephalograms were collected. The following cephalometric variables (Fig. 3) were calculated for every patient:

- FMA, the angle between the Frankfurt plane and the mandibular plane;

- MP-MxP, the angle between the mandibular plane and the maxillary plane passing through the anterior and posterior nasal spine;

- MP-OP, the angle between the mandibular plane and the occlusal plane;

- MxP-OP, the angle between the maxillary plane and the occlusal plane.

Tracings were performed by an expert operator (MT) and repeated after a two-weeks interval. An Intra-Class 


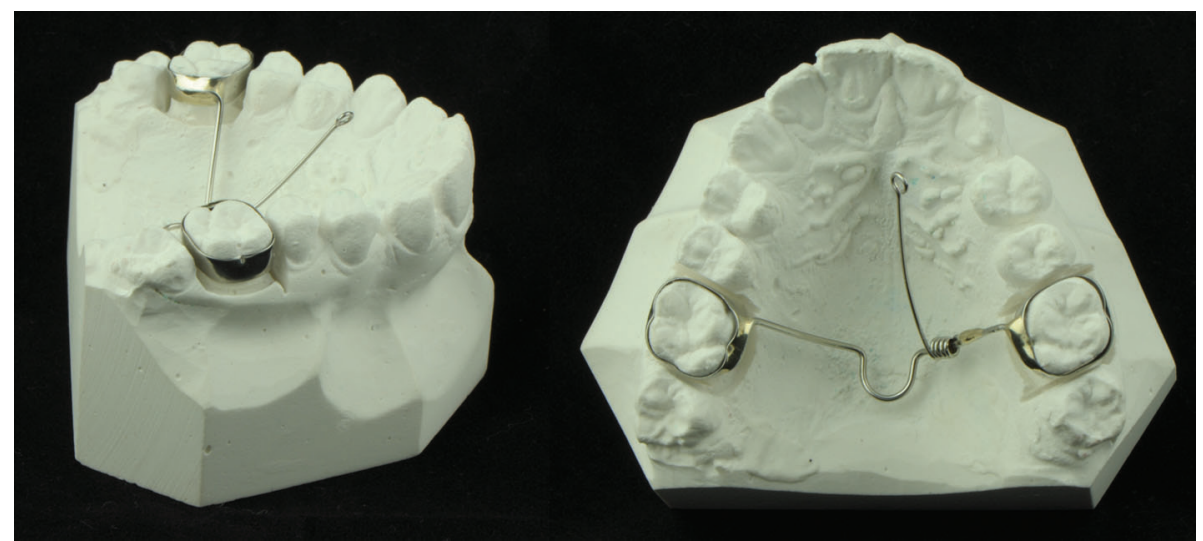

Fig. 1: The cantilever appliance used to orthodontically extrude the palatally impacted canines in the present study.
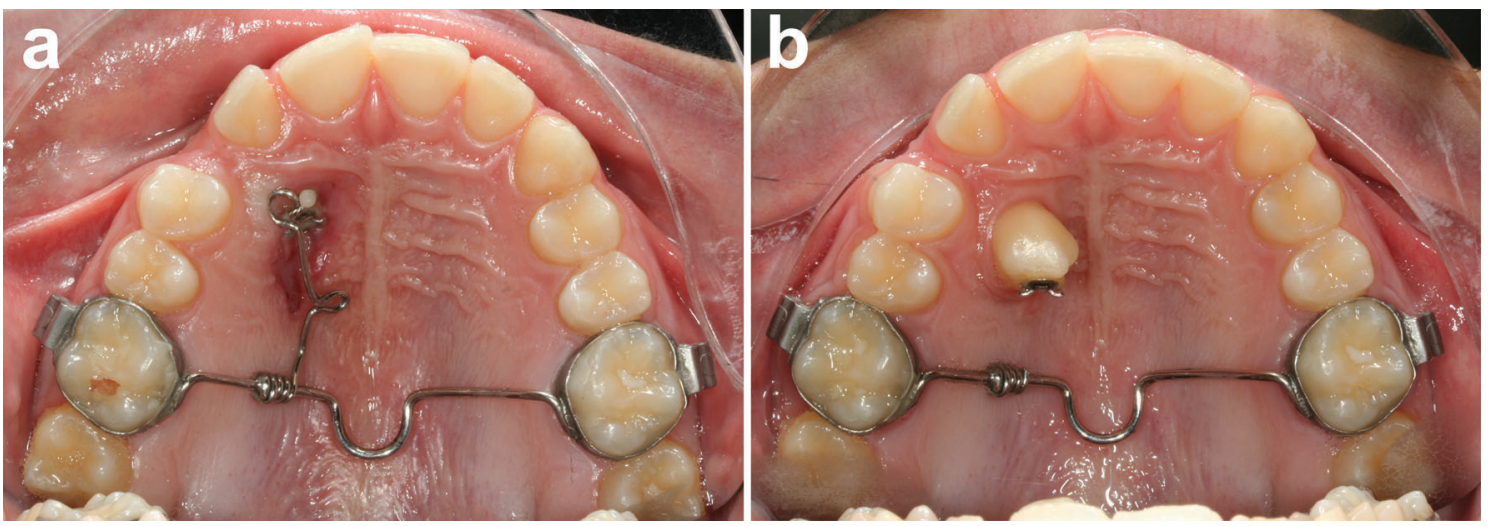

Fig. 2: An example of the cantilever appliance used to extrude an impacted canine.

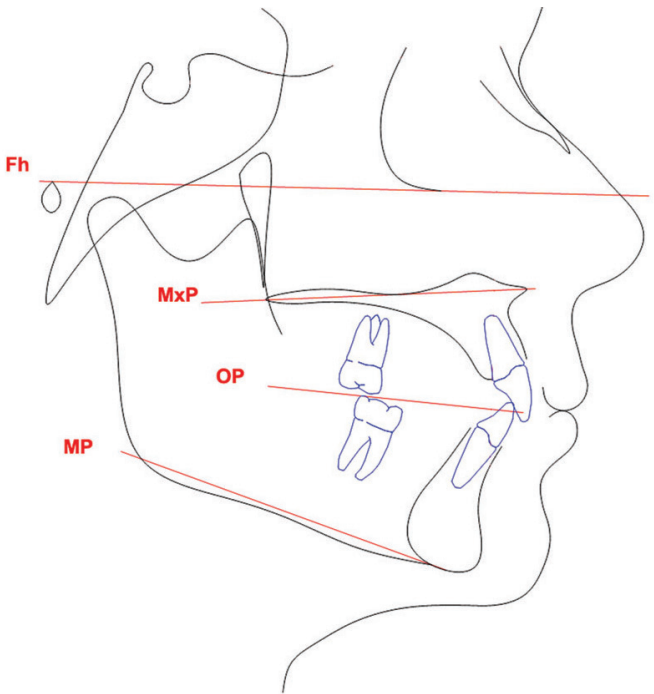

Fig. 3: Reference planes used to evaluate the facial divergence. Fh, Frankfurt plane; MxP, maxillary plane; OP, occlusal plane; MP, mandibular plane.
Correlation (ICC) coefficient was calculated between the two set of measurements to evaluate the intra-operator reliability.

\section{-Statistical Analysis}

Descriptive statistics were calculated for all the variables. A linear bivariate regression was calculated to predict orthodontic extrusion time from amount of facial divergence, as explained by the selected cephalometric angles. Normal P-P Plots were also used to check the assumption of homoscedasticity and normality of residuals. First-type error was set as 0.05. Statistical analysis was carried out using SPSS software (SPSS for Windows, Version 13.0. Chicago, SPSS Inc.).

\section{Results}

Regarding the error of the method, the calculated ICC coefficient was excellent $(>0.85)$ for all the variables, revealing good intra-observer reliability of the measurements. Descriptive statistics are reported in Table 1. According to the FMA values, 17 patients could be classified as hypo-divergent ( $65.4 \%$ of the total sample), 6 patients as normo-divergent $(23.1 \%)$, and $3(11.5 \%)$ as hyper-divergent (Fig. 4). 
Table 1: Descriptive statistics for patients' age, orthodontic extrusion time and cephalometric variables $(n=26)$.

\begin{tabular}{|l|c|c|c|}
\hline Variable & Mean & SD & $\begin{array}{c}\text { Shapiro-Wilk normality } \\
\text { test* }\end{array}$ \\
\hline Age & 15.8 & 0.9 & 0.002 \\
\hline Extrusion time & 3.6 & 0.4 & 0.086 \\
\hline FMA & 21.3 & 1.2 & $<0.001$ \\
\hline MP-MxP & 24.1 & 1.2 & 0.226 \\
\hline MP-OP & 16.4 & 0.9 & 0.238 \\
\hline MxP-OP & 7.7 & 0.8 & 0.106 \\
\hline
\end{tabular}

${ }^{*} p$ value for Shapiro-Wilk test; †expressed in months; FMA, the angle between the Frankfurt plane and the mandibular plane; MP-MxP, the angle between the mandibular plane and the maxillary plane passing through the anterior and posterior nasal spine; MP-OP, the angle between the mandibular plane and the occlusal plane; MxP-OP, the angle between the maxillary plane and the occlusal plane.

\section{Distribution of facial divergence types}

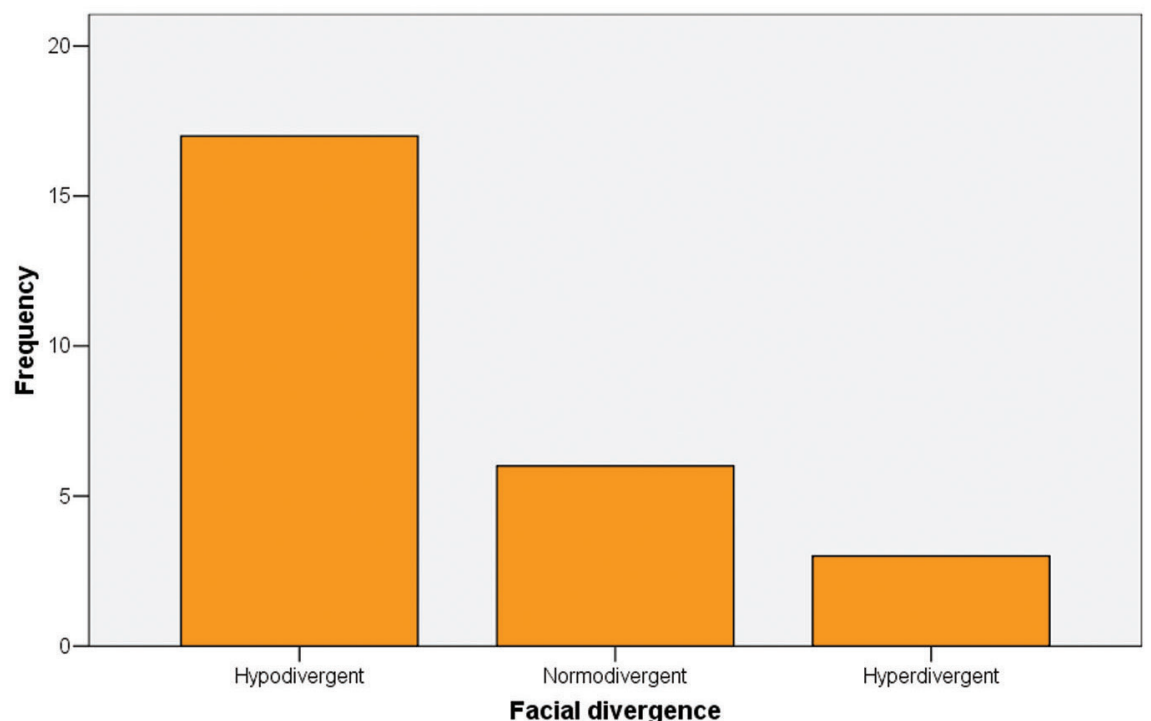

Fig. 4: Frequencies of patients classified as normodivergent, hypodivergent, or hyperdivergent according to their FMA value.

The correlation matrix for the studied variables is reported in Table 2. The variable MP-MxP was excluded from subsequent analysis because of a strong $(>0.7)$ correlation with FMA and MP-OP, to avoid multicollinearity of the data.

The linear regression model was not able to explain a relation between extrusion time and facial divergence (Table 3), therefore the null hypothesis was accepted.

\section{Discussion}

The concept of muscular anchorage, introduced in 1978 by Bench et al. (32), is of great clinical importance because orthodontists are constantly faced with it. According to this concept, the cephalometric morphology of different facial types is related to a particular muscular pattern: hypodivergent patient with strong musculature will control the teeth through muscular anchorage that 
Table 2: Correlations between orthodontic extrusion time and cephalometric variables explaining facial divergence $(\mathrm{n}=26)$.

\begin{tabular}{|l|c|c|c|c|c|}
\hline & Extrusion time & FMA & MP-MxP & Mp-OP & MxP-OP \\
\hline Extrusion time & 1 & $-0.16(0.218)$ & $-0.13(0.267)$ & $-0.04(0.422)$ & $-0.16(0.223)$ \\
\hline FMA & $-0.16(0.218)$ & 1 & $0.79(<0.001)^{*}$ & $0.65(<0.001)^{*}$ & $0.49(0.006)^{*}$ \\
\hline MP-MxP & $-0.13(0.267)$ & $0.79(<0.001)^{*}$ & 1 & $0.77(<0.001)^{*}$ & $0.67(<0.001)^{*}$ \\
\hline MP-OP & $-0.04(0.422)$ & $0.65(<0.001)^{*}$ & $0.77(<0.001)^{*}$ & 1 & $0.05(0.404)$ \\
\hline MxP-OP & $-0.16(0.223)$ & $0.49(0.006)^{*}$ & $0.67(<0.001)^{*}$ & $0.05(0.404)$ & 1 \\
\hline
\end{tabular}

Pearson correlation ( $p$ value); *statistically significant with $p<0.05$; FMA, the angle between the Frankfurt plane and the mandibular plane; MP-MxP, the angle between the mandibular plane and the maxillary plane passing through the anterior and posterior nasal spine; MP-OP, the angle between the mandibular plane and the occlusal plane; MxP-OP, the angle between the maxillary plane and the occlusal plane.

Table 3: Linear bivariate regression outcome $(\mathrm{n}=26)$.

\begin{tabular}{|l|c|c|c|c|}
\hline & $\mathbf{B}$ & $\mathbf{S E}$ & $\mathbf{p}$ & $\mathbf{V I F}$ \\
\hline $\begin{array}{l}\text { Extrusion time } \\
\text { (Constant) }\end{array}$ & $4.8^{*}$ & 1.9 & 0.025 & - \\
\hline FMA & -0.06 & 0.1 & 0.628 & 2.68 \\
\hline MP-OP & 0.04 & 0.1 & 0.811 & 2.04 \\
\hline MxP-OP & -0.05 & 0.1 & 0.770 & 1.56 \\
\hline Adjusted R & -0.09 & - & - & - \\
& & & & \\
\hline
\end{tabular}

*statistically significant with $p<0.05$; FMA, the angle between the Frankfurt plane and the mandibular plane; MP-OP, the angle between the mandibular plane and the occlusal plane; MxP-OP, the angle between the maxillary plane and the occlusal plane.

on the other hand would be less efficient in hyperdivergent patients with weak elevatory muscles (32). Clinically, this means that, for example, a greater tendency toward undesired molar extrusion would be expected as a consequence of orthodontic mechanics in hyperdivergent patients compared to hypodivergent patients, whose stronger elevatory muscles would counteract the extrusive force, or even reintrude molars (27). Several studies found different cross-sectional areas of masseter and lateral pterygoid muscles that were correlated to vertical face height $(33,34)$, and many authors observed higher biting force in hypodivergent subjects, and lower biting forces in hyperdivergent patients that were weaker than normally divergent subjects $(25,35-38)$, both in adults and in children (26). This difference in bite force is related not only to different thickness of the muscles, but also to a different mechanical advantage provided by different lever arms, constituted by different morphologies of the jaws $(33,39)$. In addition, differences in basal phosphate content of the masseter muscle correlated to facial divergence were reported: the different phosphorylation potential could reflect different levels of resting muscle tension (40). The muscles of hypodivergent patients have a higher metabolic activity at rest, keeping the bone under constant tension and maybe influencing its growth in a more horizontal pattern (40).

Facial divergence has an influence also on the position of the hyoid bone and the tongue. Hyperdivergent subjects have the tongue and the hyoid bone in a more inferior and posterior position (29), making tongue elevation difficult, resulting in reduced measured maximal tongue pressure exerted on the palatal vault (30). The pressure produced by the tongue during deglutition ranges from 41 to $709 \mathrm{~g} / \mathrm{cm} 2$ according to Winders, from 37 to 240 $\mathrm{g} / \mathrm{cm} 2$ according to Kydd and Toda (41), from 97 to 223 $\mathrm{g} / \mathrm{cm} 2$ according to Chiba et al. (28), while Xu et al. reported a mean value of $540 \mathrm{~g} / \mathrm{cm} 2$ (42). These force values exhibit no gender-related differences (30), but seem to decrease with age in elderly patients (43). During swallowing, the base of the tongue is forced upward and backward, then slides the bolus backward towards the pharynx (44); during this process, considerable force is produced against the palate, the alveolar ridge, and any orthodontic appliance across its path (42). Considering that, normally, swallowing happens 2400 times a day (45), and each event lasts slightly more than $1 \mathrm{se}-$ cond (46), it is possible to conclude that the tongue can produce tooth movements (28). This statement can be confirmed by some studies that found that a transpalatal 
arch was able to intrude the molars using the force exerted by the tongue $(47,48)$.

With all these considerations in mind, it is possible to expect an effect of tongue pressure on a device placed in the palatal vault, such as the cantilever appliance used for the present study, and that this effect could be different as the vertical skeletal pattern-and muscular pattern, as a consequence - changes. The present cantilever appliance produces forces for the extrusion of the canine

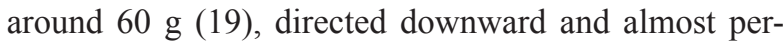
pendicular to the palatal plane. The forces produced by the tongue are considerably higher, and therefore could interfere with the forced eruption of the canine. However, results from the present study revealed that no effect on the extrusion of the canine, in terms of time needed to erupt the canine, could be explained by cephalometric variables describing the facial divergence of the patients (Table 3). This finding could be explained by the small number of severely hyperdivergent subjects in the study group, compared to normo- or hypodivergent subjects. Alternatively, the reason could be found in the position of the canine: the canine's crown and the terminal of the cantilever are located in the most anterior part of the palatal vault, and some authors reported that the pressure produced by the tongue increases as the measurement point is displaced backward, with force values measured at the level of the upper second molar being even 100 $\mathrm{g}$ higher than those measured at the level of the second premolar (28), so the tongue pressure at the level of the canine crown could be insufficient to provoke any significant effect.

On the other hand, it would be interesting to evaluate what happens to the reaction unit (i.e., the upper first molar connected to the transpalatal arch): indeed, it has been widely found that a transpalatal arch, and consequently the molars, experiences intrusive forces produced by the activity of the tongue $(28,42,48)$. At the same time, the extrusive force of the cantilever on the canine produces a reactive intrusive force on the molars, as well as a moment that results in a mesial tipping of the molars' crown (19). The two effects combined can produce a significant intrusion and tipping of the molars: this is the reason why some authors suggested the use of orthodontic miniscrews as a source of anchorage during forced eruption of maxillary canines (17). However, miniscrews are subject to a relatively high failure rate $(49,50)$ and their insertion is not always possible $(51)$. Therefore, the intrusive effects on the molar during extrusion of impacted canines will be the object of future studies.

Mention should be made of the limitations of the present study, and the principal one is surely the retrospective nature of this investigation, although care was taken to reduce the risk of selection bias by including the subjects in the study group through a rigid chronological criteria.

\section{Conclusions}

Applying a physiologic force for the orthodontic extrusion of palatally impacted canines with a specially designed cantilever appliance resulted in a mean traction time of 3.6 months. This treatment time was not explained by cephalometric variables describing the patient's divergence; therefore, palatally impacted canines can be successfully treated with the described protocol regardless of the patient's vertical skeletal and muscular pattern.

\section{References}

1. Ericson S, Kurol J. Early treatment of palatally erupting maxillary canines by extraction of the primary canines. Eur J Orthod. 1988;10:283-95.

2. Sacerdoti R, Baccetti T. Dentoskeletal features associated with unilateral or bilateral palatal displacement of maxillary canines. Angle Orthod. 2004;74:725-32.

3. Becker A, Chaushu S. Etiology of maxillary canine impaction: A review. Am J Orthod Dentofac Orthop. 2015;148:557-67.

4. Rutledge MS, Hartsfield JK. Genetic Factors in the Etiology of Palatally Displaced Canines. Semin Orthod. 2010;16:165-71.

5. Laurenziello M, Montaruli G, Gallo C, Tepedino M, Guida L, Peri1lo L, et al. Determinants of maxillary canine impaction: Retrospective clinical and radiographic study. J Clin Exp Dent. 2017;9: e1304-9.

6. Naoumova J, Kurol J, Kjellberg H. A systematic review of the interceptive treatment of palatally displaced maxillary canines. Eur $\mathrm{J}$ Orthod. 2011;33:143-9.

7. Tepedino M, Iancu-Potrubacz M, Ciavarella D, Masedu F, Marchione L, Chimenti C. Expansion of permanent first molars with rapid maxillary expansion appliance anchored on primary second molars. J Clin Exp Dent. 2018;10:e241-7.

8. Monaco A, Tepedino M, Sabetti L, Petrucci A, Sgolastra F. An adolescent treated with rapid maxillary expansion presenting with strabismus: A case report. J Med Case Rep. 2013;7:222.

9. Becker A, Chaushu S. Success rate and duration of orthodontic treatment for adult patients with palatally impacted maxillary canines. Am J Orthod Dentofac Orthop. 2003;124:509-14.

10. Stewart JA, Heo G, Glover KE, Williamson PC, Lam EW m, Major PW. Factors that relate to treatment duration for patients with palatally impacted maxillary canines. Am J Orthod Dentofac Orthop. 2001;119:216-25.

11. Baccetti T, Crescini A, Nieri M, Rotundo R, Pini Prato GP. Orthodontic treatment of impacted maxillary canines: an appraisal of prognostic factors. Prog Orthod. 2007;8:6-15.

12. Crescini A, Nieri M, Buti J, Baccetti T, Prato GPP. Orthodontic and periodontal outcomes of treated impacted maxillary canines: An appraisal of prognostic factors. Angle Orthod. 2007;77:571-7.

13. Becker A, Brin I, Ben-Bassat Y, Zilberman Y, Chaushu S. Closed-eruption surgical technique for impacted maxillary incisors: A postorthodontic periodontal evaluation. Am J Orthod Dentofac Orthop. 2002;122:9-14.

14. Bishara SE. Impacted maxillary canines : A review. Am J Orthod Dentofac Orthop. 1992;101:159-71.

15. Iancu Potrubacz M, Tepedino M, Chimenti C. Maxillary caninefirst premolar bilateral transposition in a Class III patient: A case report. Angle Orthod. 2016; 86:509-19

16. Di Palma E, Di Giuseppe B, Tepedino M, Chimenti C. Orthodontic management of bilateral maxillary caninefirst premolar transposition and bilateral agenesis of maxillary lateral incisors: A case report. Dental Press J Orthod. 2015;20:100-9.

17. Fleming PS, Sharma PK, DiBiase AT. How to...mechanically erupt a palatal canine. J Orthod. 2010;37:262-71.

18. Yadav S, Chen J, Upadhyay M, Jiang F, Roberts WE. Comparison of the force systems of 3 appliances on palatally impacted canines. Am J Orthod Dentofac Orthop. 2011;139:206-13. 
19. Tepedino M, Chimenti C, Masedu F, Iancu Potrubacz M. Predictable method to deliver physiologic force for extrusion of palatally impacted maxillary canines. Am J Orthod Dentofacial Orthop. 2018;153:195-203.

20. Iancu Potrubacz M, Chimenti C, Marchione L, Tepedino M. Retrospective evaluation of treatment time and efficiency of a predictable cantilever system for orthodontic extrusion of impacted maxillary canines. Am J Orthod Dentofac Orthop. 2018;154:55-64.

21. Mahony D. Refining occlusion with muscle balance to enhance long-term orthodontic stability. J Clin Pediatr Dent. 2005;29:93-8.

22. Di Palma E, Tepedino M, Chimenti C, Tartaglia GM, Sforza C. Effects of the functional orthopaedic therapy on masticatory muscles activity. J Clin Exp Dent. 2017;9:e886-91.

23. Di Palma E, Tepedino M, Chimenti C, Tartaglia GM, Sforza C. Longitudinal effects of rapid maxillary expansion on masticatory muscles activity. J Clin Exp Dent. 2017;9:e635-e640.

24. Pepicelli A, Woods M, Briggs C. The mandibular muscles and their importnace in orthodontics : A contemporary review. Am J Orthod Dentofac Orthop. 2005;128:774-80.

25. Proffit WR, Fields HW, Nixon WL. Occlusal Forces in Normaland Long-face Adults. J Dent Res. 1983;62:566-70.

26. García-morales P, Buschang PH, Throckmorton GS, English JD. Maximum bite force, muscle efficiency and mechanical advantage in children with vertical growth patterns. Eur J Orthod. 2003;25:265-72. 27. Spyropoulos MN, Askarieh M. Vertical control: a multifactorial problem and its clinical implications. Am J Orthod. 1976;70:70-80.

28. Chiba Y, Motoyoshi M, Namura S. Tongue pressure on loop of transpalatal arch during deglutition. Am J Orthod Dentofacial Orthop. 2003;123:29-34.

29. Urzal V, Braga AC, Ferreira AP. Hyoid bone position and vertical skeletal pattern--open bite/deep bite. Oral Health Dent Manag. 2014;13:341-7.

30. Kurabeishi H, Ryu T, Nezu M, Fukui K. Relationship between tonge pressure and maxillofacial morphology in Japanese children based on skeletal classification. J Oral Rehabil. 2018;0-1.

31. Faul F, Erdfelder E, Lang AG, Buchner A. G*Power: A flexible statistical power analysis program for the social, behavioral, and biomedical sciences. Behav Res Methods. 2007;39:175-91.

32. Bench RW, Gugino CF, Hilgers JJ. Bioprogressive therapy. Part 6. J Clin Orthod. 1978;12:123-39.

33. Raadsheerl MC, Eijden TMGJ Van, Ginkell FC Van. Contribution of Jaw Muscle Size and Craniofacial Morphology to Human Bite Force Magnitude. J Dent Res. 1999;78:31-42.

34. Weijs WA, Hillen B. Correlations between the cross-sectional area of the jaw muscles and craniofacial size and shape. Am J Phys Anthropol. 1986;70:423-31.

35. Abu Alhaija ESJ, Al Zo'ubi IA, Al Rousan ME, Hammad MM. Maximum occlusal bite forces in Jordanian individuals with different dentofacial vertical skeletal patterns. Eur J Orthod. 2010;32:71-7.

36. Ingervall B, Helkimo E. Masticatory muscle force and facial morphology in man. Arch Oral Biol. 1978;23:203-6.

37. Ciavarella D, Mastrovincenzo M, Sabatucci A, Parziale V, Granatelli F, Violante F, et al. Clinical and computerized evaluation in study of temporo-mandibular joint intracapsular disease. Minerva Stomatol. 2010;59:89-101.

38. Ciavarella D, Parziale V, Mastrovincenzo M, Palazzo A, Sabatucci A, Suriano MM, et al. Condylar position indicator and T-scan system II in clinical evaluation of temporomandibular intracapsular disease. J Craniomaxillofac Surg. 2012;40:449-55.

39. Ingervall B, Thilander B. Relation between facial morphology and activity of the masticatory muscles. J Oral Rehabil. 1974;1:131-47.

40. Al-Farra ET, Vandenborne K, Swift A, Ghafari J. Magnetic resonance spectroscopy of the masseter muscle in different facial morphological patterns. Am J Orthod Dentofac Orthop. 2001;120:42734.

41. Kydd WL, Toda JM. Tongue pressures exerted on the hard palate during swallowing. J Am Dent Assoc. 1962;65:319-30.

42. Xu K, Zeng J, Xu T. Effect of an intraoral appliance on tongue pressure measured by force exerted during swallowing. Am J Orthod Dentofac Orthop. 2016;149:55-61.

43. Utanohara Y, Hayashi ÆR, Yoshikawa ÆM. Standard Values of Maximum Tongue Pressure Taken Using Newly Developed Disposable Tongue Pressure Measurement Device. Dysphagia. 2008;23:28690.

44. Ovsenik M, Volk J, Marolt MM. A 2D ultrasound evaluation of swallowing in children with unilateral posterior crossbite. Eur J Orthod. 2014;36:665-71.

45. Straub WJ. Malfunction of the tongue Part II. The abnormal swallowing habit: its causes, effects, and results in relation to orthodontic treatment and speech therapy. Am J Orthod. 1961;47:596-617.

46. Ciavarella D, Tepedino M, Laurenziello M, Guida L, Troiano G, Montaruli G, et al. Swallowing and Temporomandibular Disorders in Adults. J Craniofac Surg. 2018;29:262-7.

47. DeBerardinis M, Stretesky T, Sinha P, Nanda RS. Evaluation of the vertical holding appliance in treatment of high-angle patients. Am J Orthod Dentofac Orthop. 2000;117:700-5.

48. Wise JB, Magness WB, Powers JM. Maxillary molar vertical control with the use of transpalatal arches. Am J Orthod Dentofacial Orthop. 1994;106:403-8.

49. Tepedino M, Masedu F, Chimenti C. Comparative evaluation of insertion torque and mechanical stability for self-tapping and self-drilling orthodontic miniscrews - an in vitro study. Head Face Med. 2017;13:10.

50. Meursinge Reynders R a, Ronchi L, Ladu L, van Etten-Jamaludin F, Bipat S. Insertion torque and success of orthodontic mini-implants: a systematic review. Am J Orthod Dentofac Orthop. 2012;142:596614.e5.

51. Tepedino M, Cattaneo PM, Masedu F, Chimenti C. Average interradicular sites for miniscrew insertion : should dental crowding be considered ? Dent Press J Orthod. 2017;22:90-7.

\section{Conflict of Interest}

The authors have declared that no conflict of interest exist. 